\title{
Singlet-Oxygen-Induced Rearrangement of Furan Derivatives
}

\author{
Nicolas Charbonnet, Emmanuel Riguet, Christian G. Bochet* \\ Department of Chemistry, University of Fribourg, Chemin du Musée 9, 1700 Fribourg, Switzerland \\ Fax +41(26)3009739; E-mail: Christian.bochet@unifr.ch
}

\begin{abstract}
Upon exposure to singlet oxygen and dimethylsulfide, the addition products between 3-furaldehydes and Grignard reagents undergo an oxidative rearrangement to give 2-substituted 3furaldehydes, in yields ranging from 26-83\%. $\mathrm{N}$-Aryl- and $\mathrm{N}$-tosylpyrroles were similarly obtained if the corresponding nitrogencontaining precursors were used instead, in equally attractive yields $(64-92 \%)$.
\end{abstract}

Key words: photochemistry, heterocycles, singlet oxygen, rearrangement, pyrroles, furans

Oxidation reactions are among the most important processes for both life and organic synthesis. The reaction between oxygen and glucose is carefully orchestrated by a series of enzymes, providing the essential form of energy for keeping organisms alive. On the other hand, the uncontrolled version of the process is the combustion reaction, which provides mainly heat and carbon dioxide. In synthesis, oxidations are rarely based on molecular oxygen, and expensive and toxic reagents are routinely used. A notable exception is the photo-oxygenation of alkenes using singlet oxygen. Essentially green and atom-economical, this process is actually one of the few industrially relevant photochemical reactions. ${ }^{1,2}$

An unprecedented Grignard addition-oxidative rearrangement sequence providing 2-alkyl-/aryl-3-furaldehydes has recently been published by Walsh et al. (Scheme 1). ${ }^{3}$ The oxidative step is induced by $N$-bromosuccinimide; we report here its photochemical counterpart.
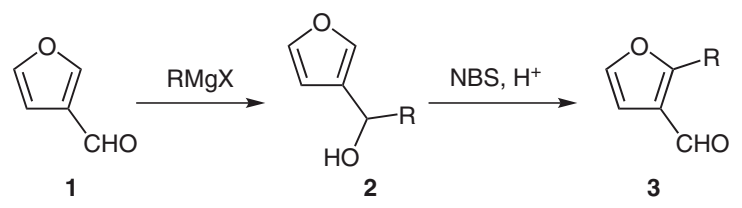

Scheme 1 Oxidative rearrangement of furans according to Walsh et al.

3-Furfural (1) is a readily available starting material, which reacts with organozinc, organolithium, and Grignard reagents to give alcohols 2, following the work of Walsh et al. ${ }^{3}$ These alcohols, if bearing electron-donating $\mathrm{R}$ side chains are quite acid-sensitive, and should not be kept for prolonged times, as already pointed out by Blechert et al. ${ }^{4}$ Exposure of $\mathbf{2 a}-\mathbf{i}$ to singlet oxygen (generated by irradiation of a saturated solution of oxygen in acetonitrile at $-40{ }^{\circ} \mathrm{C}$ in the presence of methylene blue with a $300 \mathrm{~W}$ tungsten/quartz burner light source), immediately followed by quenching at $-40{ }^{\circ} \mathrm{C}$ with dimethylsulfide and $p$-toluenesulfonic acid gave moderate to excellent yields of 2-substituted furfurals 3a-i after gradual warming up to room temperature (Scheme 2, Table 1). Low temperature during irradiation and quenching is essential to ensure optimal yields. Different conditions were screened, varying the solvent, the acid, and the reductant. Acetonitrile proved to be the best solvent (either anhydrous or containing up to $10 \%$ water), followed by dichloromethane, while hexane and methanol were found inappropriate. We suspect an attack of the latter on the endoperoxide intermediate 4 (Scheme 3 ). ${ }^{5}$ A strong acid, such as $p$-toluenesulfonic acid, was required, whereas acetic acid was inefficient. Dimethylsulfide was the only reductant that led to the desired product; phosphites, thiourea, and diphenylsulfide were unreactive. It is worth pointing out that pure oxygen was used for experimental convenience, but ambient air gave similarly good results. The nature of the R substituent has an impact on the yield; conjugated systems have a beneficial effect, whereas alkyl and strong electron-releasing groups (entry 4) lower the yields. An $N$-Boc-indolyl substituent is tolerated (entry 9), but the corresponding $N$-Boc-pyrrole led only to degradation. It is worth noting that the reaction of $\mathbf{2 h}$ resulted in the chemoselective reaction of the diene part, leading to 3h.

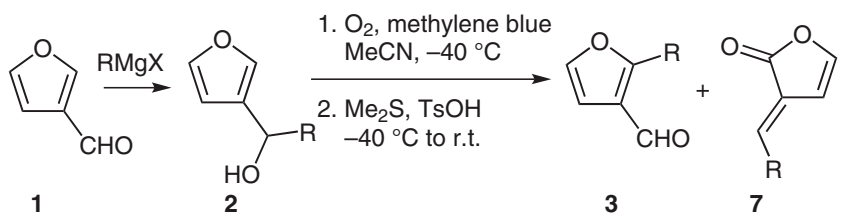

Scheme 2 Singlet-oxygen-promoted oxidative rearrangement

We propose the mechanism as shown in Scheme 3: the furan core of alcohols $\mathbf{2 a - i}$ reacts with singlet oxygen according to a very well documented process, ${ }^{6}$ leading to the endo-peroxides 4 . These peroxides are rather unstable, as proton abstraction at the anomeric site is known to give hydroxybutenolides, ${ }^{7}$ and thus should be reduced to the diols 5 before warming up the mixture. These hydroxyfurans 5 can undergo a ring opening to their enedial forms $\mathbf{6}$ and $\mathbf{6}^{\prime}$, which can re-close to $\mathbf{3}$, along the lines of the mechanism proposed by Walsh. In some cases, particular- 


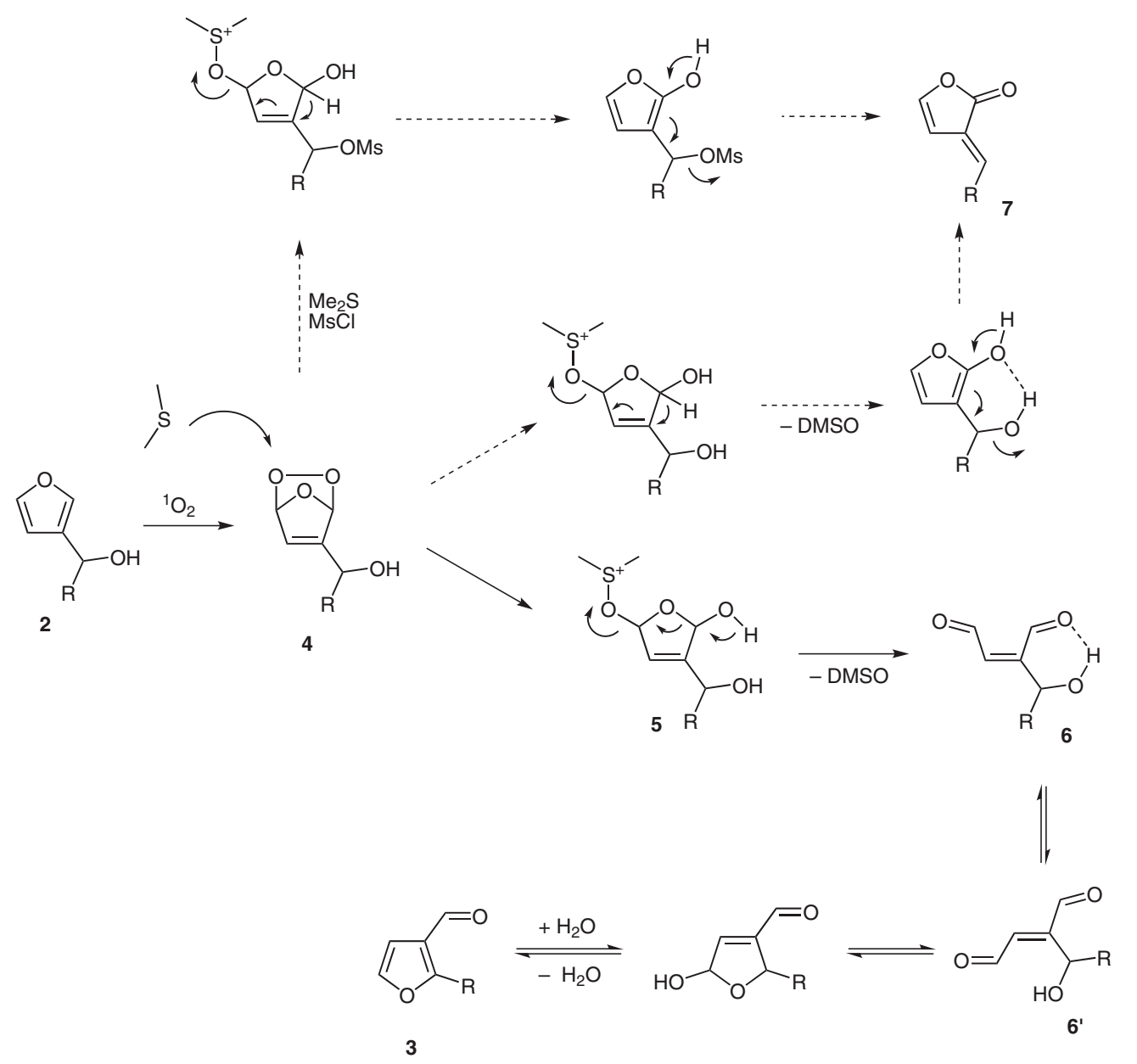

Scheme 3 Putative mechanism

Table 1 Singlet-Oxygen-Promoted Oxidative Rearrangement

\begin{tabular}{|c|c|c|c|c|}
\hline Entry & Alcohol 2 & $\mathrm{R}$ & $\begin{array}{l}\text { Yield of furan } 3 \\
(\%)^{\mathrm{a}}\end{array}$ & $\begin{array}{l}3 \text { Furanone } 7 \\
(\%)^{\mathrm{b}}\end{array}$ \\
\hline 1 & $2 a$ & $\mathrm{Ph}$ & 74 & 5 \\
\hline 2 & $2 b$ & $\mathrm{Me}$ & 40 & 6 \\
\hline 3 & $2 \mathrm{c}$ & $t-\mathrm{Bu}$ & 26 & 26 \\
\hline 4 & $2 d$ & 4- $\mathrm{MeOC}_{6} \mathrm{H}_{4}$ & 29 & 15 \\
\hline 5 & $2 \mathrm{e}$ & $n-\operatorname{Pr}$ & 38 & 8 \\
\hline 6 & $2 f$ & $4-\mathrm{F}_{3} \mathrm{CC}_{6} \mathrm{H}_{4}$ & 71 & $<2$ \\
\hline 7 & $2 \mathrm{~g}$ & $\mathrm{Bn}$ & 73 & $<2$ \\
\hline 8 & $2 \mathrm{~h}$ & vinyl $l^{\mathrm{c}}$ & 83 & $<2$ \\
\hline 9 & $2 \mathbf{i}$ & 2-N-Boc-indolyl ${ }^{\mathrm{d}}$ & 53 & $<2$ \\
\hline
\end{tabular}

${ }^{\mathrm{a}}$ Isolated yield.

${ }^{\mathrm{b}}$ Estimated by ${ }^{1} \mathrm{H}$ NMR with an internal standard.

${ }^{c}$ Overall yield based on $\mathbf{1}$.

${ }^{d}$ Prepared by the addition 2-lithio- $N$-Boc-indole to 3-ethoxycarbonylfuran, followed by reduction with $\mathrm{NaBH}_{4}$.

ly with alkyl R groups, small amounts of alkylidenefuran2-ones 7 are observed. ${ }^{8}$ The formation of this product can

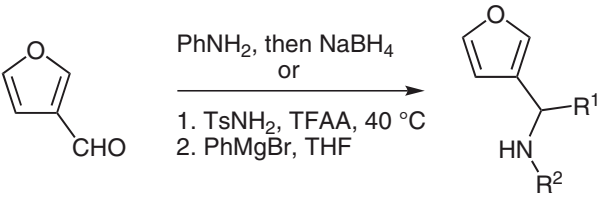

8a: $R^{1}=H, R^{2}=P h(94 \%)$ 8b: $R^{1}=P h, R^{2}=$ Ts $(74 \%)$

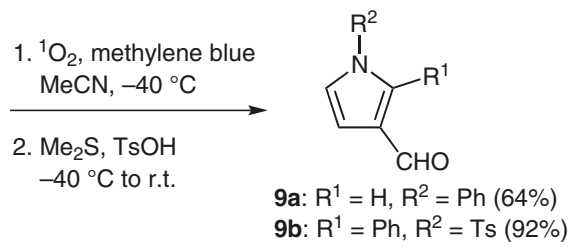

Scheme 4 Formation of pyrroles

be enhanced by the in situ treatment with a sulfonyl chloride (mesyl or tosyl); this is also compatible with a reductive cleavage of the endo-peroxides $\mathbf{4}$ into $\mathbf{7}$.

Following this mechanism, the formation of pyrrole derivatives should be also possible if the hydroxyl function of $\mathbf{2}$ is replaced with an amine. ${ }^{3}$ Thus, reductive amination of $\mathbf{1}$ with aniline gave 8a (94\%) and addition of phenylmagnesium bromide to the tosylimine derivative ${ }^{9}$ of $\mathbf{1}$ gave $\mathbf{8 b}$ 
(74\% overall). We then checked the feasibility of the process, by exposing amines 8 to the same reaction conditions (Scheme 4), and pyrroles 9 were obtained in good to excellent yields.

In summary, we reported a photochemical alternative to the halogen-induced rearrangement of 3-hydroxymethylfurans, which is also compatible with the formation of pyrroles. Applications of this chemistry in the green synthesis of industrially relevant compounds are under way.

Typical Procedure for the Oxidation with ${ }^{1} \mathrm{O}_{2}$ Followed by the Rearrangement of 3-(1'-Hydroxyalkyl)furan Derivatives

A $25 \mathrm{~mL}$, two-necked, quartz, cylindrical flask equipped with magnetic stirring bar, was charged with the corresponding 3-(1-hydroxyalkyl)furan $(1.15 \mathrm{mmol})$, methylene blue $(5.6 \mathrm{mg}, 0.013$ $\mathrm{mmol})$, and $\mathrm{MeCN}(10 \mathrm{~mL})$. The solution was cooled to $-40{ }^{\circ} \mathrm{C}$, and oxygen was bubbled gently through the solution while it was irradiated (Osram Ultra-Vitalux ${ }^{\circledR}, 300 \mathrm{~W}$ ) The consumption of 3-hydroxymethylfuran was followed by TLC $\left(\mathrm{CH}_{2} \mathrm{Cl}_{2}-\mathrm{MeOH}, 98: 2\right)$. After $90 \mathrm{~min}$, DMS $(1 \mathrm{~mL}, 13.6 \mathrm{mmol})$ was added at $-40{ }^{\circ} \mathrm{C}$, rapidly followed by PTSA ( $218 \mathrm{mg}, 1.15 \mathrm{mmol})$. The reaction mixture was allowed to warm to r.t. then stirred for $2 \mathrm{~h}$. Sat. solution of $\mathrm{Na}_{2} \mathrm{CO}_{3}$ $(30 \mathrm{~mL})$ and $\mathrm{CH}_{2} \mathrm{Cl}_{2}(50 \mathrm{~mL})$ were added. The layers were separated, the organic layer was dried over anhyd $\mathrm{MgSO}_{4}$, and concentrated under reduced pressure. The crude product was purified by flash chromatography on silica gel to afford the desired aldehyde.

\section{Acknowledgment}

This work was generously supported by the Velux foundation.

\section{References}

(1) Oelgemoeller, M.; Jung, C.; Ortner, J.; Mattay, J.; Zimmermann, E. Green Chem. 2005, 7, 35.

(2) Monnerie, N.; Ortner, J. J. Sol. Energy Eng. 2001, 123, 171.

(3) Kelly, A. R.; Kerrigan, M. H.; Walsh, P. J. J. Am. Chem. Soc. 2008, 130, 4097.

(4) Gebauer, J.; Blechert, S. Synlett 2005, 2826.

(5) Vassiliogiannakis, G.; Stratakis, M. Angew. Chem. Int. Ed. 2003, 42, 5465.

(6) For a recent review, see: Montagnon, T.; Tofi, M.; Vassiliogiannakis, G. Acc. Chem. Res. 2008, 41, 1001.

(7) (a) Fischer, M. Angew. Chem., Int. Ed. Engl. 1978, 17, 16. (b) Kernan, M. R.; Faulkner, D. J. J. Org. Chem. 1988, 53, 2773.

(8) Boukouvalas, J.; Marion, O. Synlett 2006, 1511.

(9) Lee, K. Y.; Lee, C. G.; Kim, J. N. Tetrahedron Lett. 2003, $44,1231$. 